\title{
On a more accurate half-discrete Hardy-Hilbert-type inequality related to the kernel of arc tangent function
}

\author{
Qiang Chen ${ }^{1 *}$ and Bicheng Yang ${ }^{2}$
}

${ }^{*}$ Correspondence: cq_c123@163.com 1 Department of Computer Science, Guangdong University of Education, Guangzhou 51003, Guangdong, People's Republic of China Full list of author information is available at the end of the article

\begin{abstract}
By means of weight functions and Hermite-Hadamard's inequality, and introducing a discrete interval variable, a more accurate half-discrete Hardy-Hilbert-type inequality related to the kernel of arc tangent function and a best possible constant factor is given, which is an extension of a published result. The equivalent forms and the operator expressions are also considered.
\end{abstract}

Keywords: Hardy-Hilbert-type inequality, Weight function, Hermite-Hadamard's inequality, Equivalent form, Operator

Mathematics Subject Classification: 26D15, 47A07

\section{Background}

If $p>1, \frac{1}{p}+\frac{1}{q}=1, f(x), g(y) \geq 0, f \in L^{p}\left(\mathbf{R}_{+}\right), g \in L^{q}\left(\mathbf{R}_{+}\right),\|f\|_{p}=\left(\int_{0}^{\infty} f^{p}(x) d x\right)^{\frac{1}{p}}>0$, $\|g\|_{q}>0$, then we have the following Hardy-Hilbert's integral inequality (cf. Hardy et al. 1934):

$$
\int_{0}^{\infty} \int_{0}^{\infty} \frac{f(x) g(y)}{x+y} d x d y<\frac{\pi}{\sin (\pi / p)}\|f\|_{p}\|g\|_{q}
$$

where, the constant factor $\frac{\pi}{\sin (\pi / p)}$ is the best possible. Assuming that $a_{m}, b_{n} \geq 0, a=\left\{a_{m}\right\}_{m=1}^{\infty} \in l^{p}, \quad b=\left\{b_{n}\right\}_{n=1}^{\infty} \in l^{q}, \quad\|a\|_{p}=\left(\sum_{m=1}^{\infty} a_{m}^{p}\right)^{\frac{1}{p}}>0,\|b\|_{q}>0$, we have the following Hardy-Hilbert's inequality with the same best possible constant $\frac{\pi}{\sin (\pi / p)}$ (cf. Hardy et al. 1934):

$$
\sum_{m=1}^{\infty} \sum_{n=1}^{\infty} \frac{a_{m} b_{n}}{m+n}<\frac{\pi}{\sin (\pi / p)}\|a\|_{p}\|b\|_{q} .
$$

Inequalities (1) and (2) are important in Analysis and its applications (cf. Hardy et al. 1934; Mitrinović et al. 1991; Yang 2009a, b, 2011).

In 1998, by introducing a parameter $\lambda \in(0,1]$, Yang (1998) gave an extension of (1) with the kernel $\frac{1}{(x+y)^{\lambda}}$ for $p=q=2$. Recently, Yang (2009b) gave extensions of (1) and 
(2) as follows: If $\lambda_{1}, \lambda_{2} \in \mathbf{R}, \lambda_{1}+\lambda_{2}=\lambda, k_{\lambda}(x, y)$ is a non-negative homogeneous function of degree $-\lambda$, with

$$
\begin{gathered}
k\left(\lambda_{1}\right)=\int_{0}^{\infty} k_{\lambda}(t, 1) t^{\lambda_{1}-1} d t \in \mathbf{R}_{+}, \\
\phi(x)=x^{p\left(1-\lambda_{1}\right)-1}, \psi(x)=x^{q\left(1-\lambda_{2}\right)-1}, \quad f(x), g(y) \geq 0, \\
f \in L_{p, \phi}\left(\mathbf{R}_{+}\right)=\left\{f ;|| f \|_{p, \phi}:=\left(\int_{0}^{\infty} \phi(x)|f(x)|^{p} d x\right)^{\frac{1}{p}}<\infty\right\},
\end{gathered}
$$

$g \in L_{q, \psi}\left(\mathbf{R}_{+}\right),\|f\|_{p, \phi},\|g\|_{q, \psi}>0$, then we have

$$
\int_{0}^{\infty} \int_{0}^{\infty} k_{\lambda}(x, y) f(x) g(y) d x d y<k\left(\lambda_{1}\right)|| f\left\|_{p, \phi}\right\| g \|_{q, \psi}
$$

where, the constant factor $k\left(\lambda_{1}\right)$ is the best possible. Moreover, if $k_{\lambda}(x, y)$ keeps finite value and $k_{\lambda}(x, y) x^{\lambda_{1}-1}\left(k_{\lambda}(x, y) y^{\lambda_{2}-1}\right)$ is decreasing with respect to $x>0(y>0)$, then for $a_{m}, b_{n} \geq 0$,

$$
a \in l_{p, \phi}=\left\{a ;\|a\|_{p, \phi}:=\left(\sum_{n=1}^{\infty} \phi(n)\left|a_{n}\right|^{p}\right)^{\frac{1}{p}}<\infty\right\},
$$

$b=\left\{b_{n}\right\}_{n=1}^{\infty} \in l_{q, \psi},\|a\|_{p, \phi},\|b\|_{q, \psi}>0$, we have the following Hilbert-type inequality with the same best possible constant factor $k\left(\lambda_{1}\right)$ :

$$
\sum_{m=1}^{\infty} \sum_{n=1}^{\infty} k_{\lambda}(m, n) a_{m} b_{n}<k\left(\lambda_{1}\right)\|a\|_{p, \phi}\|b\|_{q, \psi}
$$

On half-discrete Hilbert-type inequalities with the non-homogeneous kernels, Hardy et al. provided a few results in Theorem 351 of Hardy et al. (1934). But they did not prove that the the constant factors are the best possible. Yang (2005) gave an inequality with the kernel $\frac{1}{(1+n x)^{\lambda}}$ as follows:

$$
\sum_{n=1}^{\infty} \int_{0}^{\infty} \frac{a_{n} f(x)}{(1+n x)^{\lambda}} d x<B\left(\frac{\lambda}{2}, \frac{\lambda}{2}\right)\left(\sum_{n=1}^{\infty} n^{1-\lambda} a_{n}^{2} \int_{0}^{\infty} x^{1-\lambda} f^{2}(x) d x\right)^{\frac{1}{2}},
$$

and proved that the constant factor $B\left(\frac{\lambda}{2}, \frac{\lambda}{2}\right)(\lambda>0)$ is the best possible. Zhong et al. (Zhong 2008, 2011, 2012; Li and He 2007; Azar 2008; Jin and Debnath 2010; Huang 2010, 2015; Krnić and Vuković 2012; Adiyasuren et al. 2014, 2016; He 2015) investigated a few half-discrete Hilbert-type inequalities and some other Hilbert-type inequalities. In 2014, Yang et al. published a book (Yang and Debnath 2014) for building the theory of half-discrete Hilbert-type inequalities.

In this paper, by means of weight functions and Hermite-Hadamard's inequality, and introducing a discrete interval variable, a more accurate half-discrete Hardy-Hilberttype inequality related to the kernel of arc tangent function and a best possible constant 
factor is given, which is an extension of a published result mention in Yang and Debnath (2014). The equivalent forms and the operator expressions are considered.

\section{An example and some lemmas}

In the following, we make appointment that $v_{j}>0(j \in \mathbf{N}), V_{n}:=\sum_{j=1}^{n} v_{j}, 0 \leq \widetilde{v}_{n} \leq \frac{v_{n}}{2}$, $\widetilde{V}_{n}=V_{n}-\widetilde{v}_{n}, v(t):=v_{n}, t \in\left(n-\frac{1}{2}, n+\frac{1}{2}\right](n \in \mathbf{N})$, and

$$
V(y):=\int_{\frac{1}{2}}^{y} v(t) d t\left(y \in\left[\frac{1}{2}, \infty\right)\right),
$$

$p \neq 0,1, \frac{1}{p}+\frac{1}{q}=1, \delta \in\{-1,1\}, f(x), a_{n} \geq 0\left(x \in \mathbf{R}_{+}, n \in \mathbf{N}\right),\|f\|_{p, \Phi_{\delta}}=\left(\int_{0}^{\infty} \Phi_{\delta}(x) f^{p}(x)\right.$ $d x)^{\frac{1}{p}},\|a\|_{q, \widetilde{\Psi}}=\left(\sum_{n=1}^{\infty} \widetilde{\Psi}(n) b_{n}^{q}\right)^{\frac{1}{q}}$, where, $\Phi_{\delta}(x):=x^{p(1-\delta \sigma)-1}$,

$$
\widetilde{\Psi}(n):=\frac{\widetilde{V}_{n}^{q(1-\sigma)-1}}{v_{n}^{q-1}}\left(x \in \mathbf{R}_{+}, n \in \mathbf{N}\right) .
$$

Example 1 For $\rho>0,0<\sigma<\gamma \leq 1$, we set

$$
h(t):=\arctan \frac{\rho}{t^{\gamma}}\left(t \in \mathbf{R}_{+}\right)
$$

(i) Setting $u=\rho^{2} t^{-2 \gamma}$, we find

$$
\begin{aligned}
k(\sigma) & :=\int_{0}^{\infty} t^{\sigma-1} \arctan \frac{\rho}{t^{\gamma}} d t=\frac{\rho^{\sigma / \gamma}}{2 \gamma} \int_{0}^{\infty} u^{\frac{-\sigma}{2 \gamma}-1} \arctan u^{\frac{1}{2}} d u \\
& =\frac{\rho^{\sigma / \gamma}}{\sigma} \int_{0}^{\infty} \arctan u^{\frac{1}{2}} d u^{\frac{-\sigma}{2 \gamma}} \\
& =\frac{\rho^{\sigma / \gamma}}{\sigma}\left[\left.u^{\frac{-\sigma}{2 \gamma}} \arctan u^{\frac{1}{2}}\right|_{0} ^{\infty}-\frac{1}{2} \int_{0}^{\infty} \frac{u^{\frac{-\sigma}{2 \gamma}-\frac{1}{2}}}{1+u} d u\right] \\
& =\frac{\rho^{\sigma / \gamma}}{2 \sigma} \int_{0}^{\infty} \frac{u^{\left(\frac{1}{2}-\frac{\sigma}{2 \gamma}\right)-1}}{1+u} d u=\frac{\rho^{\sigma / \gamma} \pi}{2 \sigma \sin \pi\left(\frac{1}{2}-\frac{\sigma}{2 \gamma}\right)} \\
& =\frac{\rho^{\sigma / \gamma} \pi}{2 \sigma \cos \left(\frac{\pi \sigma}{2 \gamma}\right)}
\end{aligned}
$$

(ii) We obtain for $\rho>0,0<\gamma \leq 1, t>0, h(t)=\arctan \frac{\rho}{t^{\gamma}}>0$,

$$
\frac{d}{d t} h(t)=\frac{-\rho \gamma}{\left(t^{2 \gamma}+\rho^{2}\right) t^{1-\gamma}}<0, \quad \frac{d^{2}}{d t^{2}} h(t)>0 .
$$

It is evident that for $\sigma<1, t^{\sigma-1} h(t)>0$,

$$
\frac{d}{d t}\left(t^{\sigma-1} h(t)\right)<0, \quad \frac{d^{2}}{d t^{2}}\left(t^{\sigma-1} h(t)\right)>0 .
$$

(iii) Since for $n \in \mathbf{N}, V(y)>0, V^{\prime}(y)=v_{n}>0, V^{\prime \prime}(y)=0\left(y \in\left(n-\frac{1}{2}, n+\frac{1}{2}\right)\right.$, it follows that for $c>0$, we have 


$$
\begin{aligned}
h(c V(y)) V^{\sigma-1}(y) & >0, \quad \frac{d}{d y}\left(h(c V(y)) V^{\sigma-1}(y)\right)<0, \\
\frac{d^{2}}{d y^{2}}\left(h(c V(y)) V^{\sigma-1}(y)\right) & >0 \quad\left(y \in\left(n-\frac{1}{2}, n+\frac{1}{2}\right)\right)
\end{aligned}
$$

Lemma 1 If $g(t)>0, g^{\prime}(t)<0, g^{\prime \prime}(t)>0\left(t \in\left(\frac{1}{2}, \infty\right)\right)$, satisfying $\int_{\frac{1}{2}}^{\infty} g(t) d t \in \mathbf{R}_{+}$, then we have

$$
\int_{1}^{\infty} g(t) d t<\sum_{n=1}^{\infty} g(n)<\int_{\frac{1}{2}}^{\infty} g(t) d t
$$

Proof For $n_{0} \in \mathbf{N} \backslash\{1\}$, by the assumptions and Hermite-Hadamard's inequality, we have

$$
\int_{n}^{n+1} g(t) d t<g(n)<\int_{n-\frac{1}{2}}^{n+\frac{1}{2}} g(t) d t\left(n=1, \ldots, n_{0}\right) .
$$

It follows that

$$
0<\int_{1}^{n_{0}+1} g(t) d t<\sum_{n=1}^{n_{0}} g(n)<\sum_{n=1}^{n_{0}} \int_{n-\frac{1}{2}}^{n+\frac{1}{2}} g(t) d t=\int_{\frac{1}{2}}^{n_{0}+\frac{1}{2}} g(t) d t<\infty .
$$

In the same way, we still have

$$
0<\int_{n_{0}+1}^{\infty} g(t) d t \leq \sum_{n=n_{0}+1}^{\infty} g(n) \leq \int_{n_{0}+\frac{1}{2}}^{\infty} g(t) d t<\infty
$$

Hence, adding these two inequalities, we have (7).

Lemma 2 If $\rho>0,0<\sigma<\gamma \leq 1$, define the following weight coefficients:

$$
\begin{aligned}
& \omega_{\delta}(\sigma, x):=\sum_{n=1}^{\infty} \frac{x^{\delta \sigma} v_{n}}{\widetilde{V}_{n}^{1-\sigma}} \arctan \frac{\rho}{\left(x^{\delta} \widetilde{V}_{n}\right)^{\gamma}}, \quad x \in \mathbf{R}_{+}, \\
& \varpi_{\delta}(\sigma, n):=\int_{0}^{\infty} \frac{\widetilde{V}_{n}^{\sigma}}{x^{1-\delta \sigma}} \arctan \frac{\rho}{\left(x^{\delta} \widetilde{V}_{n}\right)^{\gamma}} d x, \quad n \in \mathbf{N} .
\end{aligned}
$$

We have

$$
\begin{aligned}
& \omega_{\delta}(\sigma, x)<k(\sigma)\left(x \in \mathbf{R}_{+}\right), \\
& \varpi_{\delta}(\sigma, n)=k(\sigma)(n \in \mathbf{N}),
\end{aligned}
$$

where, $k(\sigma)$ is indicated by (6). 
Proof Since

$$
\begin{aligned}
V(n) & =\int_{\frac{1}{2}}^{n+\frac{1}{2}} v(t) d t-\frac{v_{n}}{2}=V_{n}-\frac{v_{n}}{2} \\
& \leq \widetilde{V}_{n} \leq V_{n}=V\left(n+\frac{1}{2}\right)
\end{aligned}
$$

and for $t \in\left(n-\frac{1}{2}, n+\frac{1}{2}\right), V^{\prime}(t)=v_{n}$, in view of Example 1(ii)-(iii), (13), (8) and (7), we have

$$
\begin{aligned}
\frac{x^{\delta \sigma} v_{n}}{\widetilde{V}_{n}^{1-\sigma}} \arctan \frac{\rho}{\left(x^{\delta} \widetilde{V}_{n}\right)^{\gamma}} \leq \frac{x^{\delta \sigma} v_{n}}{V^{1-\sigma}(n)} \arctan \frac{\rho}{\left(x^{\delta} V(n)\right)^{\gamma}} \\
<\int_{n-\frac{1}{2}}^{n+\frac{1}{2}} \frac{x^{\delta \sigma} V^{\prime}(t)}{V^{1-\sigma}(t)} \arctan \frac{\rho}{\left(x^{\delta} V(t)\right)^{\gamma}} d t(n \in \mathbf{N}), \\
\omega_{\delta}(\sigma, x)<\sum_{n=1}^{\infty} \int_{n-\frac{1}{2}}^{n+\frac{1}{2}} \frac{x^{\delta \sigma} V^{\prime}(t)}{V^{1-\sigma}(t)} \arctan \frac{\rho}{\left(x^{\delta} V(t)\right)^{\gamma}} d t \\
=\int_{\frac{1}{2}}^{\infty} \frac{x^{\delta \sigma} V^{\prime}(t)}{V^{1-\sigma}(t)} \arctan \frac{\rho}{\left(x^{\delta} V(t)\right)^{\gamma}} d t .
\end{aligned}
$$

Setting $u=x^{\delta} V(t)$ in the above, by (6), we find

$$
\begin{aligned}
\omega_{\delta}(\sigma, x) & <\int_{0}^{x^{\delta} V(\infty)} \frac{x^{\delta \sigma} x^{-\delta}}{\left(u x^{-\delta}\right)^{1-\sigma}} \arctan \frac{\rho}{u^{\gamma}} d u \\
& \leq \int_{0}^{\infty} u^{\sigma-1} \arctan \frac{\rho}{u^{\gamma}} d u=k(\sigma) .
\end{aligned}
$$

Hence, (11) follows.

Setting $u=\widetilde{V}_{n} x^{\delta}$ in (9), we find $d u=\delta \widetilde{V}_{n} x^{\delta-1} d x$. If $\delta=1$, then

$$
\varpi_{1}(\sigma, n)=\int_{0}^{\infty} \frac{\widetilde{V}_{n}^{\sigma} \widetilde{V}_{n}^{-1}}{\left(\widetilde{V}_{n}^{-1} u\right)^{1-\sigma}} \arctan \frac{\rho}{u^{\gamma}} d u=\int_{0}^{\infty} u^{\sigma-1} \arctan \frac{\rho}{u^{\gamma}} d u
$$

if $\delta=-1$, then

$$
\varpi_{-1}(\sigma, n)=-\int_{\infty}^{0} \frac{\widetilde{V}_{n}^{\sigma} \widetilde{V}_{n}}{\left(\widetilde{V}_{n} u^{-1}\right)^{1+\sigma} u^{2}} \arctan \frac{\rho}{u^{\gamma}} d u=\int_{0}^{\infty} u^{\sigma-1} \arctan \frac{\rho}{u^{\gamma}} d u .
$$

In view of (6), we have (12).

Lemma 3 If $\rho>0,0<\sigma<\gamma \leq 1$, there exists a $n_{0} \in \mathbf{N}$, such that $v_{n} \geq v_{n+1}$ ( $\left.n \in\left\{n_{0}, n_{0}+1, \cdots\right\}\right)$, and $V_{\infty}=\infty$, then, (i) for $x \in \mathbf{R}_{+}$, we have

$$
k(\sigma)\left(1-\theta_{\delta}(\sigma, x)\right)<\omega_{\delta}(\sigma, x),
$$


where,

$$
\theta_{\delta}(\sigma, x):=\frac{1}{k(\sigma)} \int_{0}^{x^{\delta} V_{n_{0}}} u^{\sigma-1} \arctan \frac{\rho}{u^{\gamma}} d u=O\left(x^{\delta \sigma}\right) \in(0,1)
$$

(ii) for any $b>0$, we have

$$
\sum_{n=1}^{\infty} \frac{v_{n}}{\widetilde{V}_{n}^{1+b}}=\frac{1}{b}\left(\frac{1}{V_{n_{0}}^{b}}+b O(1)\right) .
$$

Proof (i) Since for $t \in(n, n+1)\left(n \geq n_{0}\right), v_{n} \geq v_{n+1}=V^{\prime}\left(t+\frac{1}{2}\right)$, by Example 1(iii) and (8), we have

$$
\begin{aligned}
\omega_{\delta}(\sigma, x) & \geq \sum_{n=n_{0}}^{\infty} \frac{x^{\delta \sigma} v_{n}}{V_{n}^{1-\sigma}} \arctan \frac{\rho}{\left(x^{\delta} V_{n}\right)^{\gamma}} \\
& =\sum_{n=n_{0}}^{\infty} \frac{x^{\delta \sigma} v_{n}}{V^{1-\sigma}\left(n+\frac{1}{2}\right)} \arctan \frac{\rho}{\left(x^{\delta} V\left(n+\frac{1}{2}\right)\right)^{\gamma}} \\
& >\sum_{n=n_{0}}^{\infty} \int_{n}^{n+1} \frac{x^{\delta \sigma} V^{\prime}\left(t+\frac{1}{2}\right)}{V^{1-\sigma}\left(t+\frac{1}{2}\right)} \arctan \frac{\rho}{\left(x^{\delta} V\left(t+\frac{1}{2}\right)\right)^{\gamma}} d t \\
& =\int_{n_{0}}^{\infty} \frac{x^{\delta \sigma} V^{\prime}\left(t+\frac{1}{2}\right)}{V^{1-\sigma}\left(t+\frac{1}{2}\right)} \arctan \frac{\rho}{\left(x^{\delta} V\left(t+\frac{1}{2}\right)\right)^{\gamma}} d t .
\end{aligned}
$$

Setting $u=x^{\delta} V\left(t+\frac{1}{2}\right)$ in the above, in view of $V_{\infty}=\infty$, by (6), we find

$$
\begin{aligned}
\omega_{\delta}(\sigma, x) & >\int_{x^{\delta} V\left(n_{0}+\frac{1}{2}\right)}^{\infty} u^{\sigma-1} \arctan \frac{\rho}{u^{\gamma}} d u \\
& =k(\sigma)-\int_{0}^{x^{\delta} V_{n_{0}}} u^{\sigma-1} \arctan \frac{\rho}{u^{\gamma}} d u=k(\sigma)\left(1-\theta_{\delta}(\sigma, x)\right), \\
\theta_{\delta}(\sigma, x) & =\frac{1}{k(\sigma)} \int_{0}^{x^{\delta} V_{n_{0}}} u^{\sigma-1} \arctan \frac{\rho}{u^{\gamma}} d u \in(0,1) .
\end{aligned}
$$

Since

$$
\arctan \frac{\rho}{u^{\gamma}} \leq \frac{\pi}{2}(u \in(0, \infty)),
$$

we find

$$
0<\theta_{\delta}(\sigma, x) \leq \frac{\pi}{2 k(\sigma)} \int_{0}^{x^{\delta} V_{n_{0}}} u^{\sigma-1} d u=\frac{\pi\left(x^{\delta} V_{n_{0}}\right)^{\sigma}}{2 \sigma k(\sigma)}
$$

and then (15) follows. 
(ii) For $b>0$, by (8), we find

$$
\begin{aligned}
\sum_{n=1}^{\infty} \frac{v_{n}}{\widetilde{V}_{n}^{1+b}} & \leq \sum_{n=1}^{n_{0}} \frac{v_{n}}{\widetilde{V}_{n}^{1+b}}+\sum_{n=n_{0}+1}^{\infty} \frac{v_{n}}{V^{1+b}(n)} \\
& \leq \sum_{n=1}^{n_{0}} \frac{v_{n}}{\widetilde{V}_{n}^{1+b}}+\sum_{n=n_{0}+1}^{\infty} \int_{n-\frac{1}{2}}^{n+\frac{1}{2}} \frac{V^{\prime}(t)}{V^{1+b}(t)} d t \\
& =\sum_{n=1}^{n_{0}} \frac{v_{n}}{\widetilde{V}_{n}^{1+b}}+\int_{n_{0}+\frac{1}{2}}^{\infty} \frac{d V(t)}{V^{1+b}(t)} \\
& =\frac{1}{b}\left(\frac{1}{V_{n_{0}}^{b}}+b \sum_{n=1}^{n_{0}} \frac{v_{n}}{\widetilde{V}_{n}^{1+b}}\right) ; \\
\sum_{n=1}^{\infty} \frac{v_{n}}{\widetilde{V}_{n}^{1+b}} & \geq \sum_{n=n_{0}}^{\infty} \frac{v_{n+1}}{V^{1+b}\left(n+\frac{1}{2}\right)} \geq \sum_{n=n_{0}}^{\infty} \int_{n}^{n+1} \frac{V^{\prime}\left(t+\frac{1}{2}\right)}{V^{1+b}\left(t+\frac{1}{2}\right)} d t \\
& =\int_{n_{0}}^{\infty} \frac{d V\left(t+\frac{1}{2}\right)}{V^{1+b}\left(t+\frac{1}{2}\right)}=\frac{1}{b V^{b}\left(n_{0}+\frac{1}{2}\right)}=\frac{1}{b V_{n_{0}}^{b}} .
\end{aligned}
$$

Hence we have (16).

Note For example, $v_{n}=\frac{1}{n^{\beta}}(n \in \mathbf{N} ; 0 \leq \beta \leq 1)$ satisfies the conditions of Lemma 3 (for $\left.n_{0}=1\right)$.

\section{Main results and operator expressions}

Theorem 1 If $\rho>0,0<\sigma<\gamma \leq 1, k(\sigma)$ is indicated by (6), then for $p>1$, $0<\|f\|_{p, \Phi_{\delta}},\|a\|_{q, \widetilde{\Psi}}<\infty$, we have the following equivalent Hardy-Hilbert-type inequalities:

$$
\begin{aligned}
I: & =\sum_{n=1}^{\infty} \int_{0}^{\infty} \arctan \frac{\rho}{\left(x^{\delta} \widetilde{V}_{n}\right)^{\gamma}} a_{n} f(x) d x<k(\sigma)|| f\left\|_{p, \Phi_{\delta}}\right\| a \|_{q, \widetilde{\Psi}}, \\
J_{1} & :=\left\{\sum_{n=1}^{\infty} \frac{v_{n}}{\widetilde{V}_{n}^{1-p \sigma}}\left[\int_{0}^{\infty} \arctan \frac{\rho}{\left(x^{\delta} \widetilde{V}_{n}\right)^{\gamma}} f(x) d x\right]^{p}\right\}^{\frac{1}{p}} \\
& <k(\sigma)\|f\|_{p, \Phi_{\delta}}, \\
J_{2} & :=\left\{\int_{0}^{\infty} \frac{1}{x^{1-q \delta \sigma}}\left[\sum_{n=1}^{\infty} \arctan \frac{\rho}{\left(x^{\delta} \widetilde{V}_{n}\right)^{\gamma}} a_{n}\right]^{q} d x\right\}^{\frac{1}{q}} \\
& <k(\sigma)\|a\|_{q, \widetilde{\Psi}} \cdot
\end{aligned}
$$


Proof By Hölder's inequality with weight (cf. Kuang 2004), we have

$$
\begin{aligned}
& {\left[\int_{0}^{\infty} \arctan \frac{\rho}{\left(x^{\delta} \widetilde{V}_{n}\right)^{\gamma}} f(x) d x\right]^{p}} \\
& =\left[\int_{0}^{\infty} \arctan \frac{\rho}{\left(x^{\delta} \widetilde{V}_{n}\right)^{\gamma}}\left(\frac{x^{\frac{1-\delta \sigma}{q}} f(x)}{\widetilde{V}_{n}^{\frac{1-\sigma}{p}}}\right)\left(\frac{\widetilde{V}_{n}^{\frac{1-\sigma}{p}}}{x^{\frac{1-\delta \sigma}{q}}}\right) d x\right]^{p} \\
& \leq \int_{0}^{\infty} \arctan \frac{\rho}{\left(x^{\delta} \widetilde{V}_{n}\right)^{\gamma}}\left(\frac{x^{\frac{p(1-\delta \sigma)}{q}} f^{p}(x)}{\widetilde{V}_{n}^{1-\sigma}}\right) d x \\
& \quad \times\left[\int_{0}^{\infty} \arctan \frac{\rho}{\left(x^{\delta} \widetilde{V}_{n}\right)^{\gamma}} \frac{\widetilde{V}_{n}^{(1-\sigma)(p-1)}}{x^{1-\delta \sigma}} d x\right]^{p-1} \\
& =\frac{\left(\varpi_{\delta}(\sigma, n)\right)^{p-1}}{\widetilde{V}_{n}^{p \sigma-1} v_{n}} \int_{0}^{\infty} \arctan \frac{\rho}{\left(x^{\delta} \widetilde{V}_{n}\right)^{\gamma}} \frac{x^{(1-\delta \sigma)(p-1)} v_{n}}{\widetilde{V}_{n}^{1-\sigma}} f^{p}(x) d x .
\end{aligned}
$$

In view of (12) and Lebesgue term by term integration theorem (cf. Kuang 2015), we find

$$
\begin{aligned}
J_{1} & \leq(k(\sigma))^{\frac{1}{q}}\left[\sum_{n=1}^{\infty} \int_{0}^{\infty} \arctan \frac{\rho}{\left(x^{\delta} \widetilde{V}_{n}\right)^{\gamma}} \frac{x^{(1-\delta \sigma)(p-1)} v_{n}}{\widetilde{V}_{n}^{1-\sigma}} f^{p}(x) d x\right]^{\frac{1}{p}} \\
& =(k(\sigma))^{\frac{1}{q}}\left[\int_{0}^{\infty} \sum_{n=1}^{\infty} \arctan \frac{\rho}{\left(x^{\delta} \widetilde{V}_{n}\right)^{\gamma}} \frac{x^{(1-\delta \sigma)(p-1)} v_{n}}{\widetilde{V}_{n}^{1-\sigma}} f^{p}(x) d x\right]^{\frac{1}{p}} \\
& =(k(\sigma))^{\frac{1}{q}}\left[\int_{0}^{\infty} \omega_{\delta}(\sigma, x) x^{p(1-\delta \sigma)-1} f^{p}(x) d x\right]^{\frac{1}{p}} .
\end{aligned}
$$

Then by (11), we have (18). By Hölder's inequality (cf. Kuang 2004), we have

$$
\begin{aligned}
I & =\sum_{n=1}^{\infty}\left[\frac{v_{n}^{\frac{1}{p}}}{\widetilde{V}_{n}^{\frac{1}{p}-\sigma}} \int_{0}^{\infty} \arctan \frac{\rho}{\left(x^{\delta} \widetilde{V}_{n}\right)^{\gamma}} f(x) d x\right]\left(\frac{\widetilde{V}_{n}^{\frac{1}{p}-\sigma} a_{n}}{v_{n}^{\frac{1}{p}}}\right) \\
& \leq J_{1}\|a\|_{q, \widetilde{\Psi}^{.}}
\end{aligned}
$$

In view of (18), we have (17). On the other hand, assuming that (17) is valid, we set

$$
a_{n}:=\frac{v_{n}}{\widetilde{V}_{n}^{1-p \sigma}}\left[\int_{0}^{\infty} \arctan \frac{\rho}{\left(x^{\delta} \widetilde{V}_{n}\right)^{\gamma}} f(x) d x\right]^{p-1}, \quad n \in \mathbf{N}
$$

Then we find $J_{1}^{p}=\|a\|_{q, \widetilde{\Psi}}^{q}$. If $J_{1}=0$, then (18) is trivially valid; if $J_{1}=\infty$, then (18) keeps impossible. Suppose that $0<J_{1}<\infty$. By (17), we have

$$
\begin{aligned}
& \|a\|_{q, \widetilde{\Psi}}^{q}=J_{1}^{p}=I<k(\sigma)\|f\|_{p, \Phi_{\delta}}\|a\|_{q, \widetilde{\Psi}}, \\
& \|a\|_{q, \widetilde{\Psi}}^{q-1}=J_{1}<k(\sigma)\|f\|_{p, \Phi_{\delta}},
\end{aligned}
$$

and then (18) follows, which is equivalent to (17). 
Still by Hölder's inequality with weight (cf. Kuang 2004), we have

$$
\begin{aligned}
& {\left[\sum_{n=1}^{\infty} \arctan \frac{\rho}{\left(x^{\delta} \widetilde{V}_{n}\right)^{\gamma}} a_{n}\right]^{q}} \\
& =\left[\sum_{n=1}^{\infty} \arctan \frac{\rho}{\left(x^{\delta} \widetilde{V}_{n}\right)^{\gamma}}\left(\frac{x^{\frac{1-\delta \sigma}{q}} v_{n}^{\frac{1}{p}}}{\widetilde{V}_{n}^{\frac{1-\sigma}{p}}}\right)\left(\frac{\widetilde{V}_{n}^{\frac{1-\sigma}{p}} a_{n}}{x^{\frac{1-\delta \sigma}{q}} v_{n}^{\frac{1}{p}}}\right)\right]^{q} \\
& \leq\left[\sum_{n=1}^{\infty} \arctan \frac{\rho}{\left(x^{\delta} \widetilde{V}_{n}\right)^{\gamma}} \frac{x^{(1-\delta \sigma)(p-1)} v_{n}}{\widetilde{V}_{n}^{1-\sigma}}\right]^{q-1} \\
& \times \sum_{n=1}^{\infty} \arctan \frac{\rho}{\left(x^{\delta} \widetilde{V}_{n}\right)^{\gamma}} \frac{\widetilde{V}_{n}^{\frac{q(1-\sigma)}{p}}}{x^{1-\delta \sigma} v_{n}^{q-1}} a_{n}^{q} \\
& =\frac{\left(\omega_{\delta}(\sigma, x)\right)^{q-1}}{x^{q \delta \sigma-1}} \sum_{n=1}^{\infty} \arctan \frac{\rho}{\left(x^{\delta} \widetilde{V}_{n}\right)^{\gamma}} \frac{\widetilde{V}_{n}^{(1-\sigma)(q-1)}}{x^{1-\delta \sigma} v_{n}^{q-1}} a_{n}^{q} .
\end{aligned}
$$

Then by (11) and Lebesgue term by term integration theorem (cf. Kuang 2015), it follows that

$$
\begin{aligned}
J_{2} & <(k(\sigma))^{\frac{1}{p}}\left\{\int_{0}^{\infty} \sum_{n=1}^{\infty} \arctan \frac{\rho}{\left(x^{\delta} \widetilde{V}_{n}\right)^{\gamma}} \frac{\widetilde{V}_{n}^{(1-\sigma)(q-1)}}{x^{1-\delta \sigma} v_{n}^{q-1}} a_{n}^{q} d x\right\}^{\frac{1}{q}} \\
& =(k(\sigma))^{\frac{1}{p}}\left\{\sum_{n=1}^{\infty} \int_{0}^{\infty} \arctan \frac{\rho}{\left(x^{\delta} \widetilde{V}_{n}\right)^{\gamma}} \frac{\widetilde{V}_{n}^{(1-\sigma)(q-1)}}{x^{1-\delta \sigma} v_{n}^{q-1}} a_{n}^{q} d x\right\}^{\frac{1}{q}} \\
& =(k(\sigma))^{\frac{1}{p}}\left\{\sum_{n=1}^{\infty} \varpi_{\delta}(\sigma, n) \frac{\widetilde{V}_{n}^{q(1-\sigma)-1}}{v_{n}^{q-1}} a_{n}^{q}\right\}^{\frac{1}{q}} .
\end{aligned}
$$

In view of (12), we have (19). By Hölder's inequality (cf. Kuang 2004), we have

$$
\begin{aligned}
I & =\int_{0}^{\infty}\left(x^{\frac{1}{q}-\delta \sigma} f(x)\right)\left[\frac{1}{x^{\frac{1}{q}-\delta \sigma}} \sum_{n=1}^{\infty} \arctan \frac{\rho}{\left(x^{\delta} \widetilde{V}_{n}\right)^{\gamma}} a_{n}\right] d x \\
& \leq\|f\|_{p, \Phi_{\delta}} J_{2} .
\end{aligned}
$$

Then by (19), we have (17). On the other hand, assuming that (19) is valid, we set

$$
f(x):=\frac{1}{x^{1-q \delta \sigma}}\left[\sum_{n=1}^{\infty} \arctan \frac{\rho}{\left(x^{\delta} \widetilde{V}_{n}\right)^{\gamma}} a_{n}\right]^{q-1}, \quad x \in \mathbf{R}_{+} .
$$

Then we find $J_{2}^{q}=\|f\|_{p, \Phi_{\delta}}^{p}$. If $J_{2}=0$, then (19) is trivially valid; if $J_{2}=\infty$, then (19) keeps impossible. Suppose that $0<J_{2}<\infty$. By (17), we have

$$
\begin{aligned}
& \|f\|_{p, \Phi_{\delta}}^{p}=J_{2}^{q}=I<k(\sigma)\|f\|_{p, \Phi_{\delta}}\|a\|_{q, \widetilde{\Psi}}, \\
& \|f\|_{p, \Phi_{\delta}}^{p-1}=J_{2}<k(\sigma)\|a\|_{q, \widetilde{\Psi}},
\end{aligned}
$$

and then (19) follows, which is equivalent to (17).

Therefore, inequalities (17), (18) and (19) are equivalent. 
Theorem 2 With regards the assumptions of Theorem 1 , if there exists a $n_{0} \in \mathbf{N}$, such that $v_{n} \geq v_{n+1}\left(n \in\left\{n_{0}, n_{0}+1, \ldots\right\}\right)$, and $V_{\infty}=\infty$, then the constant factor $k(\sigma)$ in (17), (18) and (19) is the best possible.

Proof For $\varepsilon \in(0, q \sigma)$, we set $\widetilde{\sigma}=\sigma-\frac{\varepsilon}{q}(<\min \{1, \gamma\})$, and $\widetilde{f}=\widetilde{f}(x), x \in \mathbf{R}_{+}, \widetilde{a}=\left\{\widetilde{a}_{n}\right\}_{n=1}^{\infty}$,

$$
\begin{aligned}
& \tilde{f}(x)=\left\{\begin{array}{ll}
x^{\delta(\widetilde{\sigma}+\varepsilon)-1}, & 0<x^{\delta} \leq 1 \\
0, & x^{\delta}>0
\end{array},\right. \\
& \widetilde{a}_{n}=\widetilde{V}_{n}^{\widetilde{\sigma}-1} v_{n}=\widetilde{V}_{n}^{\sigma-\frac{\varepsilon}{q}-1} v_{n}, \quad n \in \mathbf{N} .
\end{aligned}
$$

Then for $\delta= \pm 1$, we obtain

$$
\int_{\left\{x>0 ; 0<x^{\delta} \leq 1\right\}} \frac{1}{x^{1-\delta \varepsilon}} d x=\frac{1}{\varepsilon} .
$$

By (28), (16) and (14), we find

$$
\begin{aligned}
\|\widetilde{f}\|_{p, \Phi_{\delta}}\|\widetilde{a}\|_{q, \widetilde{\Psi}}=\left(\int_{\left\{x>0 ; 0<x^{\delta} \leq 1\right\}} \frac{d x}{x^{1-\delta \varepsilon}}\right)^{\frac{1}{p}}\left(\sum_{n=1}^{\infty} \frac{v_{n}}{\widetilde{V}_{n}^{1+\varepsilon}}\right)^{\frac{1}{q}} \\
\quad=\frac{1}{\varepsilon}\left(\frac{1}{V_{n_{0}}^{\varepsilon}}+\varepsilon O(1)\right)^{\frac{1}{q}}, \\
\widetilde{I}:=\int_{0}^{\infty} \sum_{n=1}^{\infty} \arctan \frac{\rho}{\left(x^{\delta} \widetilde{V}_{n}\right)^{\gamma}} \widetilde{a}_{n} \widetilde{f}(x) d x \\
=\int_{\left\{x>0 ; 0<x^{\delta} \leq 1\right\}} \sum_{n=1}^{\infty} \frac{\widetilde{V}_{n}^{\widetilde{\sigma}-1} v_{n}}{x^{1-\delta(\widetilde{\sigma}+\varepsilon)}} \arctan \frac{\rho}{\left(x^{\delta} \widetilde{V}_{n}\right)^{\gamma}} d x \\
=\int_{\left\{x>0 ; 0<x^{\delta} \leq 1\right\}} \omega_{\delta}(\widetilde{\sigma}, x) \frac{1}{x^{1-\delta \varepsilon}} d x \\
\geq k(\widetilde{\sigma}) \int_{\left\{x>0 ; 0<x^{\delta} \leq 1\right\}}\left(1-O\left(x^{\delta\left(\sigma-\frac{\varepsilon}{q}\right)}\right)\right) \frac{1}{x^{1-\delta \varepsilon}} d x \\
=k(\widetilde{\sigma})\left[\int_{\left\{x>0 ; 0<x^{\delta} \leq 1\right\}} \frac{d x}{x^{1-\delta \varepsilon}}-\int_{\left\{x>0 ; 0<x^{\delta} \leq 1\right\}} O\left(\frac{1}{x^{1-\delta\left(\sigma+\frac{\varepsilon}{p}\right)}}\right) d x\right] \\
=\frac{1}{\varepsilon} k\left(\sigma-\frac{\varepsilon}{q}\right)\left(1-\varepsilon O_{1}(1)\right) .
\end{aligned}
$$

If there exists a positive constant $K \leq k(\sigma)$, such that (17) is valid when replacing $k(\sigma)$ to $K$, then in particular, we have $\varepsilon \widetilde{I}<\varepsilon K \mid \widetilde{f}\left\|_{p, \Phi_{\delta}}\right\| \widetilde{a} \|_{q, \widetilde{\Psi}}$, namely,

$$
k\left(\sigma-\frac{\varepsilon}{q}\right)\left(1-\varepsilon O_{1}(1)\right)<K\left(\frac{1}{V_{n_{0}}^{\varepsilon}}+\varepsilon O(1)\right)^{\frac{1}{q}} .
$$

It follows that $k(\sigma) \leq K\left(\varepsilon \rightarrow 0^{+}\right)$. Hence, $K=k(\sigma)$ is the best possible constant factor of (17). 
The constant factor $k(\sigma)$ in (18) [(19)] is still the best possible. Otherwise, we would reach a contradiction by (22) [(25)] that the constant factor in (17) is not the best possible.

For $p>1$, we find $\widetilde{\Psi}^{1-p}(n)=\frac{v_{n}}{\widetilde{V}_{n}^{1-p \sigma}}(n \in \mathbf{N}), \Phi_{\delta}^{1-q}(x)=\frac{1}{x^{1-q \delta \sigma}}\left(x \in \mathbf{R}_{+}\right)$, and define the following real normed spaces:

$$
\begin{aligned}
L_{p, \Phi_{\delta}}\left(\mathbf{R}_{+}\right) & =\left\{f ; f=f(x), x \in \mathbf{R}_{+},\|f\|_{p, \Phi_{\delta}}<\infty\right\}, \\
l_{q, \widetilde{\Psi}} & =\left\{a ; a=\left\{a_{n}\right\}_{n=1}^{\infty},\|a\|_{q, \widetilde{\Psi}}<\infty\right\}, \\
L_{q, \Phi_{\delta}^{1-q}}\left(\mathbf{R}_{+}\right) & =\left\{h ; h=h(x), x \in \mathbf{R}_{+},\|h\|_{q, \Phi_{\delta}^{1-q}}<\infty\right\}, \\
l_{p, \widetilde{\Psi}^{1-p}} & =\left\{c ; c=\left\{c_{n}\right\}_{n=1}^{\infty},\|c\|_{p, \widetilde{\Psi}^{1-p}}<\infty\right\} .
\end{aligned}
$$

Assuming that $f \in L_{p, \Phi_{\delta}}\left(\mathbf{R}_{+}\right)$, setting

$$
c=\left\{c_{n}\right\}_{n=1}^{\infty}, \quad c_{n}:=\int_{0}^{\infty} \arctan \frac{\rho}{\left(x^{\delta} \widetilde{V}_{n}\right)^{\gamma}} f(x) d x, \quad n \in \mathbf{N},
$$

we can rewrite (18) as ||$c||_{p, \widetilde{\Psi}^{1-p}}<k(\sigma)|| f \|_{p, \Phi_{\delta}}<\infty$, namely, $c \in l_{p, \widetilde{\Psi}^{1-p}}$.

Definition 1 Define a half-discrete Hardy-Hilbert-type operator $T_{1}: L_{p, \Phi_{\delta}}\left(\mathbf{R}_{+}\right) \rightarrow l_{p, \widetilde{\Psi}^{1-p}}$ as follows: For any $f \in L_{p}, \Phi_{\delta}\left(\mathbf{R}_{+}\right)$, there exists a unique representation $T_{1} f=c \in l_{p, \widetilde{\Psi}^{1-p}}$. Define the formal inner product of $T_{1} f$ and $a=\left\{a_{n}\right\}_{n=1}^{\infty} \in l_{q, \widetilde{\Psi}}$ as follows:

$$
\left(T_{1} f, a\right):=\sum_{n=1}^{\infty}\left[\int_{0}^{\infty} \arctan \frac{\rho}{\left(x^{\delta} \widetilde{V}_{n}\right)^{\gamma}} f(x) d x\right] a_{n} .
$$

Then we can rewrite (17) and (18) as follows:

$$
\begin{aligned}
& \left(T_{1} f, a\right)<k(\sigma)|| f \mid\left\|_{p, \Phi_{\delta}}\right\| a \|_{q, \widetilde{\Psi}}, \\
& \left\|T_{1} f\right\|_{p, \widetilde{\Psi}^{1-p}}<k(\sigma)|| f \|_{p, \Phi_{\delta}} .
\end{aligned}
$$

Define the norm of operator $T_{1}$ as follows:

$$
\left\|T_{1}\right\|:=\sup _{f(\neq \theta) \in L_{p, \Phi_{\delta}}\left(\mathbf{R}_{+}\right)} \frac{\left\|T_{1} f\right\|_{p, \widetilde{\Psi}^{1-p}}}{\|f\|_{p, \Phi_{\delta}}} .
$$

Then by (32), it follows that $\left\|T_{1}\right\| \leq k(\sigma)$. Since by Theorem 2 , the constant factor in (32) is the best possible, we have

$$
\left\|T_{1}\right\|=k(\sigma)=\frac{\rho^{\sigma / \gamma} \pi}{2 \sigma \cos \left(\frac{\pi \sigma}{2 \gamma}\right)} .
$$

Assuming that $a=\left\{a_{n}\right\}_{n=1}^{\infty} \in l_{q, \widetilde{\Psi}}$, setting 


$$
h(x):=\sum_{n=1}^{\infty} \arctan \frac{\rho}{\left(x^{\delta} \widetilde{V}_{n}\right)^{\gamma}} a_{n}, \quad x \in \mathbf{R}_{+},
$$

we can rewrite (19) as $\|h\|_{q, \Phi_{\delta}^{1-q}}<k(\sigma)\|a\|_{q, \widetilde{\Psi}}<\infty$, namely, $h \in L_{q, \Phi_{\delta}^{1-q}}\left(\mathbf{R}_{+}\right)$.

Definition 2 Define a half-discrete Hardy-Hilbert-type operator $T_{2}: l_{q, \widetilde{\Psi}} \rightarrow L_{q, \Phi_{\delta}^{1-q}}\left(\mathbf{R}_{+}\right)$ as follows: For any $a=\left\{a_{n}\right\}_{n=1}^{\infty} \in l_{q, \widetilde{\Psi}}$, there exists a unique representation $T_{2} a=h \in L_{q, \Phi_{\delta}^{1-q}}\left(\mathbf{R}_{+}\right)$. Define the formal inner product of $T_{2} a$ and $f \in L_{p, \Phi_{\delta}}\left(\mathbf{R}_{+}\right)$as follows:

$$
\left(T_{2} a, f\right):=\int_{0}^{\infty}\left[\sum_{n=1}^{\infty} \arctan \frac{\rho}{\left(x^{\delta} \widetilde{V}_{n}\right)^{\gamma}} a_{n}\right] f(x) d x .
$$

Then we can rewrite (17) and (19) as follows:

$$
\begin{aligned}
& \left(T_{2} a, f\right)<\left.k(\sigma)\|f\|\right|_{p, \Phi_{\delta}}\|a\|_{q, \widetilde{\Psi}}, \\
& \left\|T_{2} a\right\|_{q, \Phi_{\delta}^{1-q}}<k(\sigma)\|a\| \|_{q, \widetilde{\Psi}} .
\end{aligned}
$$

Define the norm of operator $T_{2}$ as follows:

$$
\left\|T_{2}\right\|:=\sup _{a(\neq \theta) \in l_{q, \widetilde{\Psi}}} \frac{\left\|T_{2} a\right\|_{q, \Phi_{\delta}^{1-q}}}{\|a\|_{q, \widetilde{\Psi}}} .
$$

Then by (36), we find $\left\|T_{2}\right\| \leq k(\sigma)$. Since by Theorem 2, the constant factor in (36) is the best possible, we have

$$
\left\|T_{2}\right\|=k(\sigma)=\frac{\rho^{\sigma / \gamma} \pi}{2 \sigma \cos \left(\frac{\pi \sigma}{2 \gamma}\right)}=\left\|T_{1}\right\|
$$

Remark (i) For $\delta=-1$ in (17), we obtain the following inequality with the homogeneous kernel of degree 0 :

$$
\sum_{n=1}^{\infty} \int_{0}^{\infty} \arctan \left(\frac{\rho x^{\gamma}}{\widetilde{V}_{n}^{\gamma}}\right) a_{n} f(x) d x<\frac{\rho^{\sigma / \gamma} \pi}{2 \sigma \cos \left(\frac{\pi \sigma}{2 \gamma}\right)}\|f\|_{p, \Phi_{-1}}\|a\|_{q, \widetilde{\Psi}} .
$$

(ii) For $\delta=1$ in (17), we obtain the following inequality with the non-homogeneous kernel:

$$
\sum_{n=1}^{\infty} \int_{0}^{\infty} \arctan \frac{\rho}{\left(x \widetilde{V}_{n}\right)^{\gamma}} a_{n} f(x) d x<\frac{\rho^{\sigma / \gamma} \pi}{2 \sigma \cos \left(\frac{\pi \sigma}{2 \gamma}\right)} \mid f f\left\|_{p, \Phi_{1}}\right\| a \|_{q, \widetilde{\Psi}}
$$


(iii) For $\widetilde{\mu}_{n}=0(n \in \mathbf{N})$ in (17), we have the following inequality:

$$
\sum_{n=1}^{\infty} \int_{0}^{\infty} \arctan \frac{\rho}{\left(x^{\delta} V_{n}\right)^{\gamma}} a_{n} f(x) d x<\frac{\rho^{\sigma / \gamma} \pi}{2 \sigma \cos \left(\frac{\pi \sigma}{2 \gamma}\right)}\|f\|\left\|_{p, \Phi_{\delta}}\right\| a \|_{q, \Psi},
$$

where, the constant factor $\frac{\rho^{\sigma / \gamma} \pi}{2 \sigma \cos \left(\frac{\pi \sigma}{2 \gamma}\right)}$ is still the best possible. Hence, inequality (17) is a more accurate form of (40) (for $0<\widetilde{\mu}_{n} \leq \frac{\mu_{n}}{2}, n \in \mathbf{N}$ ).

(iv) For $\mu_{n}=1\left(x \in \mathbf{R}_{+}, n \in \mathbf{N}\right), \delta=-1$ in (40), we have the following inequality:

$$
\begin{aligned}
& \sum_{n=1}^{\infty} a_{n} \int_{0}^{\infty} \arctan \rho\left(\frac{x}{n}\right)^{\gamma} f(x) d x \\
& =\int_{0}^{\infty} f(x) \sum_{n=1}^{\infty} \arctan \rho\left(\frac{x}{n}\right)^{\gamma} a_{n} d x \\
& <\frac{\rho^{\sigma / \gamma} \pi}{2 \sigma \cos \left(\frac{\pi \sigma}{2 \gamma}\right)}\left[\int_{0}^{\infty} x^{p(1+\sigma)-1} f^{p}(x) d x\right]^{\frac{1}{p}}\left[\sum_{n=1}^{\infty} n^{q(1-\sigma)-1} b_{n}^{q}\right]^{\frac{1}{q}},
\end{aligned}
$$

which is a particular case of Example 3.2 in Yang and Debnath (2014) for $\lambda=0, \lambda_{1}=-\sigma, \lambda_{2}=\sigma$ and $k_{\lambda}(x, n)=\arctan \rho\left(\frac{x}{n}\right)^{\gamma}$.

We still can obtain some inequalities in Theorems 1-4, by using some particular parameters.

\section{Conclusion}

By means of the technique of real analysis, weight functions and Hermite-Hadamard's inequality, and introducing a discrete interval variable and parameters, a more accurate half-discrete Hardy-Hilbert-type inequality related to the kernel of arc tangent function and a best possible constant factor is given. The equivalent forms and the operator expressions are also considered. The method of weight functions is very important, which is the key to help us proving the main results with the best possible constant factor. The lemmas and theorems provide an extensive account of this type of inequalities.

Authors' contributions

BY carried out the mathematical studies, participated in the sequence alignment and drafted the manuscript. QC participated in the design of the study and performed the numerical analysis. Both authors read and approved the final manuscript.

\section{Author details}

1 Department of Computer Science, Guangdong University of Education, Guangzhou 51003, Guangdong, People's Republic of China. ${ }^{2}$ Department of Mathematics, Guangdong University of Education, Guangzhou 51003, Guangdong, People's Republic of China.

\section{Acknowledgements}

This work is supported by Science and Technology Planning Project of Guangdong Province (No. 2013A011403002), and Appropriative Researching Fund for Professors and Doctors, Guangdong University of Education (No. 2015ARF25). We are grateful for their help.

\section{Competing interests}

The authors declare that they have no competing interests.

Received: 8 June 2016 Accepted: 22 July 2016

Published online: 11 August 2016 
References

Adiyasuren V, Batbold Ts, Krnić M (2014) Half-discrete Hilbert-type inequalities with mean operators, the best constants, and applications. Appl Math Comput 231:148-159

Adiyasuren V, Batbold Ts, Krnić M (2016) Multiple Hilbert-type inequalities involving some differential operators. Banach J Math Anal 10(2):320-337

Azar LE (2008) On some extensions of Hardy-Hilbert's inequality and applications. J Inequal Appl 2008:1-14, Article ID 546829

Hardy GH, Littlewood JE, Pólya G (1934) Inequalities. Cambridge University Press, Cambridge

He B (2015) A multiple Hilbert-type discrete inequality with a new kernel and best possible constant factor. J Math Anal Appl 431:902-990

Huang Q (2015) A new extension of Hardy-Hilbert-type inequality. J Inequal Appl 2015:397

Huang Q, Yang B (2010) On a multiple Hilbert-type integral operator and applications. J Inequal Appl 2009:1-13, Article ID 192197

Jin J, Debnath L (2010) On a Hilbert-type linear series operator and its applications. J Math Anal Appl 371(2):404-691

Krnić M, Vuković P (2012) On a multidimensional version of the Hilbert-type inequality. Anal Math 38:291-303

Kuang J (2004) Applied inequalities. Shangdong Science Technic Press, Jinan

Kuang J (2015) Real and functional analysis (continuation), vol 2. Higher Education Press, Beijing

Li Y, He B (2007) On inequalities of Hilbert's type. Bull Aust Math Soc 76(1):1-13

Mitrinović DS, Pečarić JE, Fink AM (1991) Inequalities involving functions and their integrals and derivatives. Kluwer Academic Publishers, Boston

Yang B (1998) On Hilbert's integral inequality. J Math Anal Appl 220:778-785

Yang B (2005) A mixed Hilbert-type inequality with a best constant factor. Int J Pure Appl Math 20(3):319-328

Yang B (2009a) Hilbert-type integral inequalities. Bentham Science Publishers Ltd., Sharjah

Yang B (2009b) The norm of operator and Hilbert-type inequalities. Science Press, Beijing

Yang B (2011) Discrete Hilbert-type inequalities. Bentham Science Publishers Ltd., Sharjah

Yang B, Debnath L (2014) Half-discrete Hilbert-type inequalities. World Scientific Publishing, Singapore

Zhong W (2008) The Hilbert-type integral inequalities with a homogeneous kernel of $-\lambda$-degree. J Inequal Appl 2008:1-12, Article ID 917392

Zhong W (2011) A mixed Hilbert-type inequality and its equivalent forms. J Guangdong Univ Educ 31(5):18-22

Zhong W (2012) A half discrete Hilbert-type inequality and its equivalent forms. J Guangdong Univ Educ 32(5):8-12

\section{Submit your manuscript to a SpringerOpen ${ }^{\circ}$ journal and benefit from:}

Convenient online submission

\section{- Rigorous peer review}

- Immediate publication on acceptance

- Open access: articles freely available online

- High visibility within the field

- Retaining the copyright to your article

Submit your next manuscript at $\boldsymbol{\nabla}$ springeropen.com 